\title{
Dynamical Parton Distributions at NNLO
}

\section{Pedro JIMENEZ-DELGADO*}

TU Dortmund, Institut für Physik, 44221 Dortmund, Germany

E-mail: pjimenez@het.physik.uni-dortmund.de

Utilizing recent DIS measurements and Drell-Yan data we determine at NNLO (3-loop) of QCD the dynamical parton distribution functions of the nucleon generated radiatively from valencelike positive input distributions at an optimally chosen low resolution scale. These are compared with "standard" NNLO distributions generated from positive input distributions at some fixed and higher resolution scale. Although the NNLO corrections imply in both approaches an improved value of $\chi^{2}$, present DIS data are still not sufficiently accurate to distinguish between NLO results and the minute NNLO effects of a few percent, despite of the fact that the dynamical NNLO uncertainties are somewhat smaller than the NLO ones and both are, as expected, smaller than those of their "standard" counterparts. The dynamical predictions for the longitudinal structure function of the proton become perturbatively stable already at $Q^{2}=2-3 \mathrm{GeV}^{2}$ where precision measurements could even delineate NNLO effects in the very small-x region. This is in contrast to the common "standard" approach but NNLO/NLO differences are here less distinguishable due to the much larger uncertainty bands.

European Physical Society Europhysics Conference on High Energy Physics

July 16-22, 2009

Krakow, Poland

\footnotetext{
* Speaker.
} 
The parton distribution functions of the nucleon are usually extracted from experimental data by two essentially different approaches which differ in their choice of the parametrizations of the input distributions at some low input scale $Q_{0}^{2}$. In the common approach used by most other groups, hereafter referred to as "standard", the input scale is fixed at some arbitrarily chosen $Q_{0}^{2} \geq 1$ $\mathrm{GeV}^{2}$, and the corresponding input distributions are unrestricted, allowing even for negative gluon distributions in the small Bjorken- $x$ region (i.e., negative cross-sections like $F_{\mathrm{L}}$ ). Alternatively the parton distributions at $Q^{2}>1 \mathrm{GeV}^{2}$ are QCD radiatively (dynamically) generated from valencelike (i.e., positive definite, $x f>0$ with $x f \rightarrow 0$ for $x \rightarrow 0$ ) input distributions for all partons at an optimally determined input scale $Q_{0}^{2}<1 \mathrm{GeV}^{2}$. This more restrictive ansatz implies more predictive power and less uncertainties concerning the behavior of the parton distributions in the small- $x$ region at $Q^{2}>Q_{0}^{2}$, which is to a large extent due to QCD dynamics $\left(Q^{2}\right.$-evolution).

In this talk we briefly overview some of the results encountered in the (first) determination of the dynamical parton distribution functions of the nucleon at NNLO of QCD as presented in [1], where details of the analysis and further necessary references have been given. For consistency reasons we only include in our analysis deep inelastic scattering (DIS) and Drell-Yan dimuon production data, for which all required theoretical NNLO ingredients (except the ones for heavy quark production) are available. Heavy quark flavors have been treated within the "fixed flavor number scheme" (FFNS) where, besides the gluon, only the light quark flavors $(u, d, s)$ are considered massless partons within the nucleon. This factorization scheme is fully predictive in the heavy quark $(c, b, t)$ sector, where the full heavy quark mass dependence is taken into account in the production cross-sections as required experimentally, in particular in the threshold region. Our parametrization of the input distributions [1] depends on 21 free parameters, 13 of which (including $\alpha_{s}\left(M_{Z}^{2}\right)$ ) are also included in the error analysis; this follows the usual Hessian method with a tolerance parameter $T \simeq 4.5$ [1]. Within this framework we have carried out a dynamical (dyn) analysis, which optimal input scale turned out to be $Q_{0}^{2}=0.55 \mathrm{GeV}^{2}$, and a "standard" (std) fit which input scale was fixed to $Q_{0}^{2} \equiv 2 \mathrm{GeV}^{2}$. This allows us to compare the features of both approaches and to test the the dependence in model assumptions.

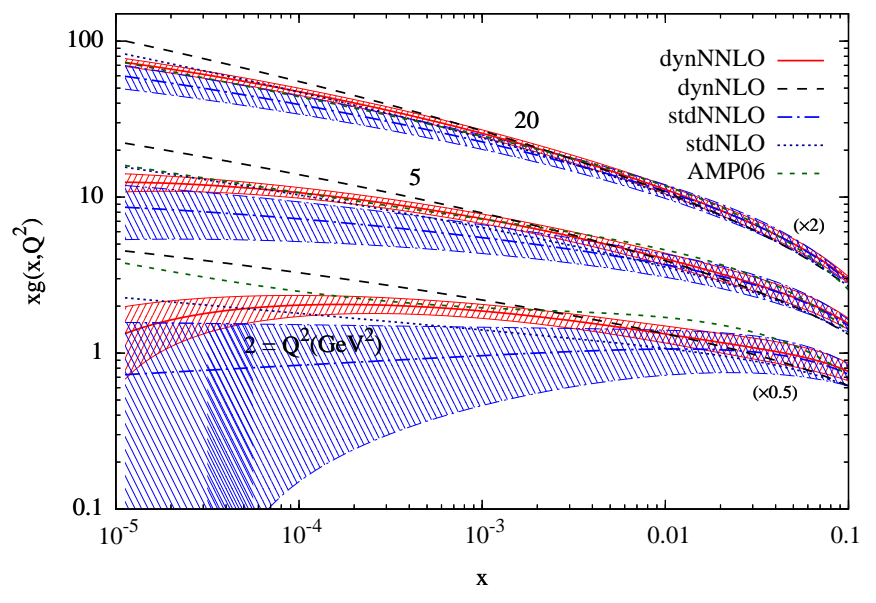

Figure 1: Dynamical and "standard" NNLO gluon distributions at small $x$. [1] 
As a first illustration of our dyn/std results we quote the values obtained for $\alpha_{s}\left(M_{Z}^{2}\right)$ at NNLO:

$$
\alpha_{s}^{\mathrm{dyn}}\left(M_{Z}^{2}\right)=0.1124 \pm 0.0020, \quad \alpha_{s}^{\mathrm{std}}\left(M_{Z}^{2}\right)=0.1158 \pm 0.0035
$$

these are in approximate agreement with the results of other analyses; note however that the differences in central values obtained in different analyses are usually larger than the estimated uncertainties, in particular fits to DIS data usually yield smaller values than other global analyses [1,2]. The uncertainty of our dynamical result is about half as large as the "standard" one. Apart from the larger evolution distance, this is due to the strongly constrained valence-like input gluon distribution in the small- $x$ region; consequently the energy-momentum sum rule constrains it sufficiently in the medium to large $x$-region, which is not the case for "standard" input gluons. This distinctive valence-like gluon input at low $Q^{2}<1 \mathrm{GeV}^{2}$ implies a far stronger constrained gluon distribution at larger values of $Q^{2}$ as compared to a gluon density obtained from a "standard" fit with a conventional non-valence-like input at $Q^{2}>1 \mathrm{GeV}^{2}$, as can be seen in Fig. 1. Notice that this "standard" NNLO input gluon distribution at $Q_{0}^{2}=2 \mathrm{GeV}^{2}$ is also compatible with a valence-like small- $x$ behavior. The uncertainties generally decrease as $Q^{2}$ increases due to the $\mathrm{QCD} Q^{2}$-evolution, but the ones of the dynamical predictions in the small- $x$ region remain substantially smaller than the uncertainties of the "standard" results.

As mentioned before, due to our valence-like input, the dynamical small- $x$ results $\left(x \lesssim 10^{-2}\right)$ for physical observables are predictions being mainly generated by QCD. This is in contrast to "standard" results where the gluon and sea input distributions do not vanish as $x \rightarrow 0$ and therefore the finite small $-x$ behavior of the input gluon and sea distributions is here fine tuned to reproduce the data. The more restrictive ansatz of the dynamical model usually leads to marginally larger $\chi^{2}$ for the statistically most significant data sets included in the fits, for example $\chi_{\text {dyn }}^{2}=0.90$ as compared to $\chi_{\text {std }}^{2}=0.87$ for the DIS data included in our analyses [1]; for the same reasons it fits comparably (or better) data with lower significance. In any case, besides this completely marginal differences in $\chi^{2}$, the dynamical approach to parton distributions does not have any disadvantage with respect to "standard" ones, while its predictive power is an important and desirable feature.

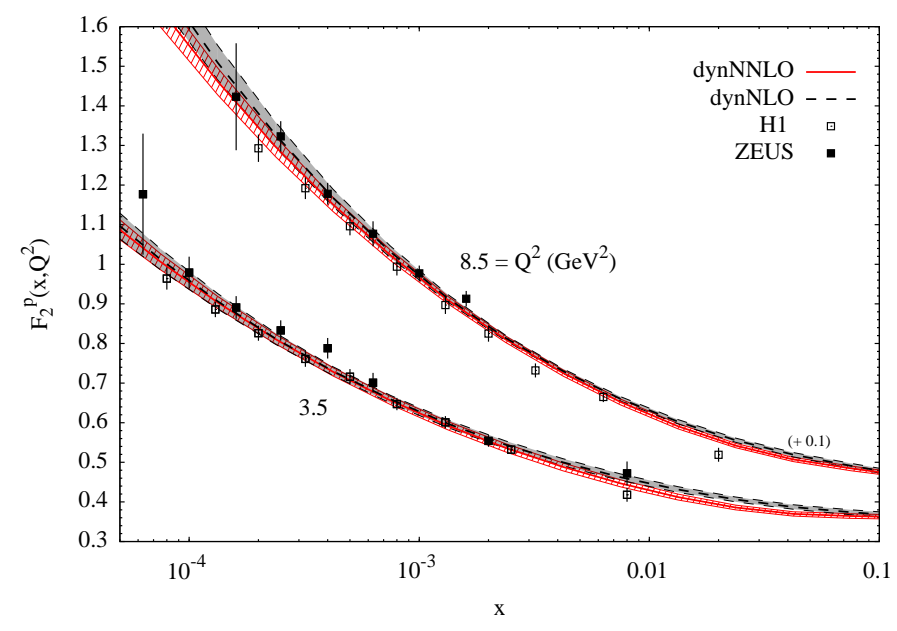

Figure 2: Typical uncertainty bands of our dynamical NNLO and NLO results for $F_{2}\left(x, Q^{2}\right)$. [1] 

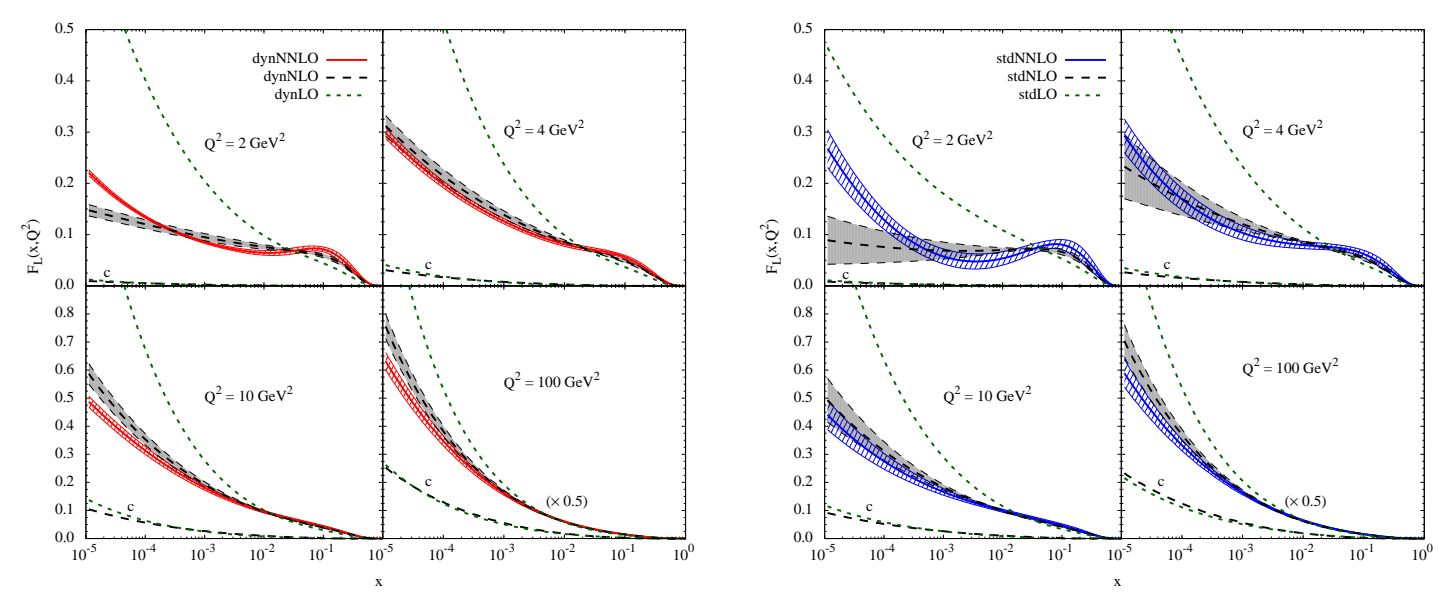

Figure 3: Dynamical/“standard” results for $F_{L}\left(x, Q^{2}\right)$. [1]

Turning now to our results for DIS structure functions, we note that we have explicitly used for our analysis the experimentally directly measured "reduced" cross-sections, which (for not too large values of $Q^{2}$ ) is dominated by the one-photon exchange cross-section $\sigma_{r}=F_{2}-\left(y^{2} / Y_{+}\right) F_{L}$; where $y=\frac{Q^{2}}{x s}$ and $Y_{+}=1+(1-y)^{2}$. Although the inclusion of NNLO corrections imply an improved value of $\chi^{2}$ (typically $\chi_{\mathrm{NNLO}}^{2} \simeq 0.9 \chi_{\mathrm{NLO}}^{2}$ ), and a reduction of the uncertainties, present high precision DIS data are not sufficiently accurate to distinguish between the NLO results [3] and the minute NNLO effects of a few percent. This is illustrated in Fig. 2, where the experimental errors for the dominant $F_{2}\left(x, Q^{2}\right)$ are far bigger than the differences between the NLO and NNLO results. Moreover, theoretical uncertainties of the analyses (such as factorization schemes, higher-twists and QED contributions) are comparable in size to the NNLO effects [4].

The effects of $F_{L}$ become increasingly relevant as $x$ decreases at a given $Q^{2}$, where $y$ increases. This is seen in the data as a flattening of the growth of $\sigma_{r}\left(x, Q^{2}\right)$ as $x$ decreases to very small values at fixed $Q^{2}$, leading eventually to a turnover since $F_{L}$ is positive; as is the case for all our dyn/std NNLO (as well as NLO/LO) results, which are in good agreement with all HERA measurements [1]. The perturbative stability of our predictions for the longitudinal cross-section is illustrated in Fig. 3. The dynamical predictions become stable already at $Q^{2} \gtrsim 3 \mathrm{GeV}^{2}$, where the gluon contribution becomes dominant and where precision measurements could even delineate NNLO effects in the very small- $x$ region. Within the common "standard" approach the absolute values of the NNLO and NLO results differ more at smaller $Q^{2}$ than the dynamical ones, but the differences between the NNLO and NLO results are here less distinguishable due to the larger uncertainties.

\section{References}

[1] P. Jimenez-Delgado and E. Reya, Phys. Rev. D79 (2009) 074023.

[2] J. Blümlein, Proceedings of DIS 2007, Vol. 2 (2007) 1099; arXiv:0706.2430 [hep-ph].

[3] M. Glück, P. Jimenez-Delgado and E. Reya, Eur. Phys. J. C53 (2008) 355.

[4] M. Glück, E. Reya and C. Schuck, Nucl. Phys. B754 (2006) 178. 